TITLE:

\title{
Surface modification of islets with PEG-lipid for improvement of graft survival in intraportal transplantation.
}

\section{$\operatorname{AUTHOR}(\mathrm{S}):$}

Teramura, Yuji; Iwata, Hiroo

\section{CITATION:}

Teramura, Yuji ... [et al]. Surface modification of islets with PEG-lipid for improvement of graft survival in intraportal transplantation.. Transplantation 2009, 88(5): 624-630

\section{ISSUE DATE:}

2009-09-15

URL:

http://hdl.handle.net/2433/87308

\section{RIGHT:}

c 2009 Lippincott Williams \& Wilkins, Inc. 許諾条件により本文は201010-01に公開。 
Surface modification of islets with PEG-lipid for improvement of graft survival in intraportal transplantation

Yuji Teramura ${ }^{1,3}$ and Hiroo Iwata ${ }^{2}$

1 Department of Nano-Medicine Merger Education Unit, and Department of Polymer Chemistry, Graduate School of Engineering, Kyoto University, 2 Institute for Frontier Medical Sciences, Kyoto University, 53 Kawara-cho, Shogoin, Sakyo-ku, Kyoto, 6068507, Japan.

Key words: bioartificial pancreas; islets; poly(ethylene glycol)-lipid (PEG-lipid); intraportal transplantation; graft survival

Word count: Text-3364 words, Abstract-235 words

Tables and Figures: 5 Figures including 2 color Figures and 1 Table

${ }^{3}$ Address correspondence and reprint requests to Yuji Teramura, Ph.D.

E-mail: teramura@frontier.kyoto-u.ac.jp, Tel: +81-75-751-4115, Fax: +81-75-751-4116 
This study was supported in part by CREST and JST, and by a Grant-in-Aid for

Scientific Research (A) (No. 18200034, 21240051) from the Ministry of Education,

Culture, Sports, Science and Technology (MEXT) of Japan and the Ministry of Health, Labor and Welfare of Japan (H20-007).

All authors make a declaration of no conflict of interest.

Author Contributions:

${ }^{1}$ specific contributions to the work are research design, the writing of the paper, the performance of the research, and data analysis

${ }^{2}$ specific contributions to the work are research design and the writing of the paper

\section{Current address}

${ }^{1}$ Radioisotope Research (RI) Center, Kyoto University, Yoshida-Konoe-Cho, Sakyo-ku, Kyoto, 606-8501, Japan.

${ }^{2}$ Department of Reparative Materials, Institute for Frontier Medical Sciences, Kyoto University, 53 Kawara-Cho, Shogoin, Sakyo-ku, Kyoto, 606-8507, Japan. 


\section{Abbreviations:}

islet, islet of Langerhans; FBS, fetal bovine serum; PEG, poly(ethylene glycol); PBS, phosphate-buffered saline; ELISA, enzyme-linked immunosorbent assay; HE, hematoxylin-eosin; DPPE, Dipalmitoyl-sn-glycerol-3-phosphatidylethanolamine; STZ, streptozotocin; MEM, minimum essential medium; NHS-PEG-Mal, $\alpha-N-$ Hydroxysuccinimidyl- $\omega$-maleimidyl poly(ethylene glycol) 


\section{Abstract}

Background: Transplantation of islets of Langerhans (islets) is a promising technique for treating insulin-dependent diabetes mellitus (type I). One unsolved issue is the early graft loss due to inflammatory reactions triggered by blood coagulation and complement activation that occurs immediately after transplantation into the liver through the portal vein. Several proposed approaches for improvement of the graft survival include heparin coating and covalent poly(ethylene glycol) (PEG) conjugation. We previously have studied the improvement of graft survival by modification of islet surfaces using amphiphilic PEG-conjugated phospholipid and bioactive molecules. Here we analyzed the effect of PEG-modification on the improvement of graft survival immediately after intraportal transplantation into streptozotocin-induced diabetic mice.

Methods: The surface of hamster islets was modified with PEG-lipid. PEG-lipid modified islets (PEG-islets) were transplanted into the liver through the portal vein of streptozotocin-induced diabetic mice. We measured the graft survival periods and blood insulin levels immediately after intraportal transplantation to determine the cell damage to islets. Histocytochemical analyses of liver were also performed post intraportal transplantation. 
Results: The graft survival of PEG-islets was significantly prolonged compared with bare islets in livers of diabetic mice. Reduction of blood insulin level within 60 min after transplantation of PEG-islets suggests that the cell damage observed immediately after transplantation could be suppressed by surface modification with PEG in comparison with bare islets.

Conclusion: Our approach for the improvement of graft survival will be useful in the clinical setting. 


\section{Introduction}

Although transplantation of islets of Langerhans (islets) is still an experimental procedure, this approach has been proven to be a safe and effective method for treating patients with insulin-dependent diabetes mellitus (type I). Notably, the success achieved with the Edmonton protocol has established clinical islet transplantation as an alternative to pancreas transplantation [1, 2]. However, some aspects of islet transplantation remain to be improved, including increase of islet isolation efficiency, improvement in islet preservation, increase in the efficacy of immunosuppressive drug dosage protocols, and reduction of islet loss in the early phase following transplantation. Among these issues, graft loss poses the most serious limitations for current islet transplantation protocols. Transplantation of islets from a single donor pancreas is not sufficient to achieve normoglycemia in a patient [2], suggesting that many islets are lost soon after intraportal transplantation.

Innate immune reactions are involved in the destruction of islets exposed to fresh blood in the portal vein [3-7]. Blood coagulation and complement systems are activated upon exposure of islets to fresh blood components; chemotactic factors, tissue factor, chemokines, and other inflammatory mediators are released during this early phase, which induce instant blood-mediated inflammatory reactions resulting in graft loss [5]. 
Some approaches to regulate early coagulation and blood-mediated inflammatory reactions, such as administration of Melagatran [6] and dextran sulfate [7], have been studied, and suppression of these reactions has improved transplantation success by reducing islet loss in animal models. However, it is difficult to apply these methods to the clinical situation because systemic administration is associated with a risk of bleeding. Several methods have been reported for improvement of graft survival in the liver, such as coating of islet surface with polymers and bioactive molecules $[8,9]$. Contreras et al. reported the improvement of graft survival in the liver by covalent conjugation of poly(ethylene glycol) to the islet surface and transfection of an apoptosis suppressing gene [8]. Recently, Nilsson et al. proposed a new method for preventing instant blood-mediated inflammatory reactions by coating islets with heparin to inhibit surface thrombosis formation following transplantation [9].

We previously studied the effect of surface modification of living cells and islets using amphiphilic polymers such as PEG-conjugated phospholipid and poly(vinyl alcohol) with alkyl side chains on the improvement of graft survival during cell transplantation [10-14]. The results from our methods are promising, as PEG-lipid derivatives can be immobilized to the cell surface via hydrophobic interactions between the lipid bilayer 
membrane. Furthermore, a thin PEG layer can form on the islet surface without cytotoxicity and volume increase.

In this paper, we evaluated the efficacy of PEG-modification on the improvement of graft survival immediately after transplantation in the liver through the portal vein of streptozotocin-induced diabetic mice. We predicted that surface modification with PEG could suppress the coagulation reactions and inflammatory reactions that mediate islet loss during the early phase of intraportal transplantation. We studied the graft survival periods, performed histochemical analysis and measured blood insulin levels immediately after intraportal transplantation to determine the cell damage to islets. 


\section{Materials and methods}

\section{Materials}

$\alpha-N$-Hydroxysuccinimidyl- $\omega$-maleimidyl poly(ethylene glycol) (NHS-PEG-Mal, Mw: 5000) was purchased from Nektar Therapeutics (San Carlos, USA). We purchased 1, 2Dipalmitoyl-sn-glycerol-3-phosphatidylethanolamine (DPPE) from NOF Corporation (Tokyo, Japan). Dichloromethane, chloroform, N, N'-dimethylformamide (DMF), diethyl ether, streptozotocin (STZ) and tri-sodium citrate dehydrate were obtained from Nacalai Tesuque (Kyoto, Japan). ICN Biomedicals Inc (Aurora, OH) supplied cystein. Fluorescein isothiocyanate (FITC) and Hoechst 33342 were purchased from Dojindo Laboratories (Kumamoto, Japan). Alexa 488-labeled goat anti-guinea pig IgG, minimum essential medium (MEM), HEPES buffer solution and Medium 199 were purchased from Invitrogen Co. (Carlsbad, USA). Fetal bovine serum (FBS) was obtained from BioWest (Miami, USA), and phosphate-buffered saline (PBS) from Nissui Pharmaceutical Co. Ltd. (Tokyo, Japan). Enzyme-linked immunosorbent assay (ELISA) kits for the insulin assay were from Shibayagi Co., Ltd. (Gunma, Japan). Tissue-Tek was purchased from Sakura Finetechnical Co. Ltd. (Tokyo, Japan). Goat normal serum and polyclonal guinea pig anti-insulin were obtained from Dako (Glostrup, Denmark). We purchased 10\% Formalin solution and Triton X-100 from 
Wako Pure Chemical (Osaka, Japan). Isoflurene was purchased from Abbott Japan Co., Ltd. (Japan).

\section{Synthesis of PEG-conjugated phospholipid (PEG-lipid)}

Mal-PEG-lipid was synthesized by first dissolving NHS-PEG-Mal NHS-PEG-Mal (180 mg) and DPPE (20 mg) in chloroform and stirring for $24 \mathrm{~h}$ at RT. After precipitation with diethyl ether, Mal-PEG-lipid was obtained as a white powder (190 mg, yield 80\%). ${ }^{1} \mathrm{H}-\mathrm{NMR}\left(\mathrm{CDCl}_{3}, 400 \mathrm{MHz}, \delta \mathrm{ppm}\right): 0.88\left(\mathrm{t}, 6 \mathrm{H},-\mathrm{CH}_{3}\right), 1.25\left(\mathrm{br}, 56 \mathrm{H},-\mathrm{CH}_{2^{-}}\right) 3.64(\mathrm{br}$, 480H, PEG), 6.71 (s, 2H, -HC=CH-, maleimide).

In this experiment, the maleimide group was previously deactivated by the addition of cystein. FITC-labeled PEG-lipid [12], which has a similar structure and activity as PEG-lipid, was visualized in islets by confocal laser scanning microscopy (FLUOVIEW FV500, Olympus, Tokyo, Japan).

\section{Preparation of PEG-lipid-modified islets}

Islets were isolated from pancreases of Syrian hamsters (7-8 weeks, females, Japan SLC, Inc., Shizuoka, Japan) by collagenase digestion [15]. The islets were cultured for 4 days after isolation to remove or sediment cells that were damaged during the isolation 
procedure; cells were cultured in Medium 199 with 10\% FBS, 8.8 mM HEPES buffer, 100 units/mL penicillin, $100 \mu \mathrm{g} / \mathrm{mL}$ streptomycin, and $8.8 \mathrm{U} / \mathrm{mL}$ heparin. A PEG-lipid solution (500 $\mu \mathrm{g} / \mathrm{mL}$ in $100 \mu \mathrm{L}$ of MEM) was added to islets suspended in serum-free MEM, and the mixture was incubated for $1 \mathrm{~h}$ at RT. After washing with serum-free MEM, PEG-lipid-modified islets were transplanted into the livers of diabetic mice.

\section{Static insulin secretion test by glucose stimulation}

Static insulin secretion tests were performed using 100 PEG-islets to evaluate their ability to secrete insulin in response to changes in glucose concentration. Bare islets were used as a control. Islets were exposed to $0.1 \mathrm{~g} / \mathrm{dl}$, then $0.3 \mathrm{~g} / \mathrm{dl}$, and finally $0.1 \mathrm{~g} / \mathrm{dl}$ of glucose in Krebs-Ringer solution for 1 hour each at $37^{\circ} \mathrm{C}$. The solutions were collected after the one-hour incubation in each glucose concentration, and insulin concentrations were determined in each solution by ELISA.

\section{Transplantation of PEG-islets into livers of STZ-induced diabetic mice}

Balb/c mice were used as recipients of islets and PEG-islets. Diabetes was induced in Balb/c mice (6 weeks, males, Japan SLC, Inc.) by intraperitoneal injection of STZ (230 $\mathrm{mg} / \mathrm{kg}$ body weight in citrate buffer, $\mathrm{pH} 4.5$ ) ten days prior to transplantation. An 
animal was considered diabetic when its plasma glucose level exceeded $400 \mathrm{mg} / \mathrm{dl}$ in two consecutive measurements.

PEG-islets or control islets (500 or 1000 islets) were transplanted into the liver through the portal vein of STZ-induced diabetic mice, which were anesthetized during surgery by mask inhalation of Isoflurane using a specialized instrument (400 Anesthesia Unit; Univentor, Malta); the Isoflurane concentration was 4.5-5.0 \% induction and 2.0 for maintenance with airflow rate of $200 \mathrm{ml} / \mathrm{min}$. Diabetic mice were treated with either 500 or 1000 control islets or PEG-islets. Mice were housed in cages with free access to food and water. Non-fasting plasma glucose levels were measured using a glucose sensor (DIAmeter- $\alpha$ glucocard; Arkray, Kyoto, Japan) between 11 a.m. and 1 p.m. before and after transplantation. Blood samples were taken from the subclavian vein. Graft failure was defined as two consecutive plasma glucose level determinations 200 mg/dL. All animal experiments were approved by the Kyoto University Animal Care Committee.

\section{Histochemical analysis}

Mice were sacrificed after transplantation of islets or PEG-islets into the liver through portal vein of diabetic mice for $1 \mathrm{hr}$ or 1 day. Livers were removed, washed with saline, 
immersed in $10 \%$ formalin solution, and incubated for 2 days at RT. The formalin solution was removed and the livers were sequentially maintained in $3 \%$ and $10 \%$ sucrose in PBS for 2 days, followed by incubation in 20\% sucrose in PBS for 2 days at RT. Livers were then embedded in Tissue-Tek for freezing. The frozen specimens were sliced (6- $\mu \mathrm{m}$ thick) by a cryostat (CM 3050S IV, Leica, Solms, Germany) and sections were permeabilized with $0.2 \%$ Triton $\mathrm{X}-100$ in PBS at RT for $15 \mathrm{~min}$. The samples were then treated with $10 \%$ normal goat serum in PBS for $1 \mathrm{~h}$ to block non-specific binding of antibodies. Sections were treated with 1\% polyclonal guinea pig anti-insulin in PBS containing 3\% goat normal serum for $3.5 \mathrm{hr}$ at RT, followed by washing with PBS. The samples were then incubated with fluorescent labeled secondary antibody, 0.2\% Alexa 488 Goat anti-guinea pig IgG in PBS containing 3\% goat normal serum at RT for 1.5 h. Cell nuclei were counterstained with Hoechst 33342. The stained sections were analyzed using fluorescence microscopy (BX51, Olympus Optical Co. Ltd., Tokyo, Japan). The sliced sections were also stained with hematoxylin-eosin (HE) using a conventional staining method.

Determination of insulin levels in blood after intraportal transplantation of PEGislets 
After transplantation of control or PEG-islets (1000 islets) into the liver of diabetic mice through the portal vein, blood was taken from the subclavian vein at 15, 30, 60 min and centrifuged to obtain plasma. Hamster insulin in the plasma of mice was determined by ELISA. 


\section{Results}

\section{Surface modification of islets with PEG-lipid}

We previously studied the modification of islet surfaces with amphiphilic polymers such as PEG-conjugated phospholipid and poly(vinyl alcohol) with alkyl side chains for the improvement of graft survival [10-14]. Our method is promising for intraportal transplantation of islet as no damage to cell function was observed and no volume increase of islets was detected after modification of islet surface.

In this study, we used amphiphilic PEG-lipid (shown in Fig. 1a) for modification of islet surfaces and predicted that the surface modification with PEG could suppress the inflammatory and coagulation reactions that mediate islet loss immediately after intraportal transplantation. The maleimide group at the end of the PEG chain can be used to immobilize bioactive molecules to the islet surface. Our aim was to evaluate the efficacy of PEG on islet surfaces to improve graft survival, therefore the maleimide group was deactivated by cystein prior to surface modification of islets. Islets were treated with a mixture of FITC-labeled PEG-lipid and PEG-lipid, and clearly detectable fluorescence was observed at the periphery of islets, indicating that the PEG chain was incorporated on the surface of islets (Fig. 1b). Furthermore, the hydrophobic lipid portion of PEG-lipid was observed to be spontaneously incorporated into the lipid 
bilayer membrane of islets. These results indicate that PEG-lipid can form a layer on the islet surface without apparent damage to islet morphology.

\section{Insulin secretion by glucose stimulation}

Next we examined the ability of PEG-islets to control insulin release in response to glucose level changes using a glucose stimulation test; the results are summarized in Fig. 2. No significant difference in insulin release was observed between control islets and PEG-islets. When the glucose concentration was increased from $0.1 \mathrm{~g} / \mathrm{dL}$ to $0.3 \mathrm{~g} / \mathrm{dL}$, both control and PEG-islets increased insulin release compared to basal levels. When islets were re-exposed to $0.1 \mathrm{~g} / \mathrm{dL}$, insulin release returned to basal levels. These results indicate that the PEG-lipid surface modification did not affect the ability of islets to regulate and release insulin.

\section{Intraportal transplantation into liver of STZ-induced diabetic mice}

We next evaluated the efficacy of PEG-modification on the improvement of graft survival immediately after intraportal transplantation. Hamster islets were transplanted into the livers of STZ-induced diabetic mice through the portal vein. This transplantation is a concordant xenotransplantation model. Recipient mice were not 
treated with immuno-suppressive therapy. Figure 3 shows changes in non-fasting blood glucose levels of recipient mice after intraportal transplantation of either 500 or 1000 bare islets or PEG-islets. In this xenotransplantation model, the blood glucose level normalized after transplantation of islets; however, levels returned to hyperglycemic levels around 7 days because the grafts were lost by immune rejection in recipient mice. In the mice transplanted with 500 islets (Fig. 3a), the blood glucose levels in all mice except one were not normalized 1 day after transplantation. Normoglycemia was achieved in 4 of 9 mice at 3 days and blood glucose levels were maintained for several days. At 7 days post-transplantation, most mice returned to hyperglycemic states. These results indicated that induction of islet cell damage occurred immediately after transplantation into the liver, and some of islets were destroyed, resulting in loss of function. The blood glucose level could not be properly regulated by the remaining islets during the early phase following post transplantation. Upon transplantation of 1000 islets (Fig. 3b), normoglycemia was achieved in all mice at 2 days and continued until grafts were rejected (around 7 days). In contrast, when 500 PEG-islets were transplanted, 5 of 6 mice were normoglycemic at 2 days and this was maintained until the graft was rejected (around 7 days). On transplantation of 1000 PEG-islets, all mice were normoglycemic at 2 days, and this was also maintained until the graft was rejected 
(around 7 days). The period of graft survival in recipients of control and PEG-islets transplantation are summarized in Table 1 . We found a significant difference between the period of graft survival upon transplantation of 500 control and PEG-islets. These results indicate that the islet damage that occurs immediately after transplantation into the liver could be suppressed by surface modification of islets with PEG-lipids. The period of graft survival was prolonged, although the immune rejection reaction could not be suppressed. On the other hand, we did not observe any significant difference in graft survival between recipients of 1000 control and PEG-islets. We speculate that mice transplanted with 500 control islets did not receive sufficient islets, as most were destroyed after transplantation and blood glucose levels could not be controlled. On the other hand, in mice transplanted with 500 PEG-islets, there were sufficient islets remaining, as cell damage to islets could be suppressed by surface modification with PEG-lipid; furthermore, blood glucose levels could be normalized, indicating the improvement of graft survival. For mice transplanted with 1000 control islets, there were sufficient islets remaining to control blood glucose levels, despite the loss of some islets. These results suggested that it is possible to suppress complement activation and blood coagulation-mediated inflammatory reactions that induce cell damage to islets 
immediately after transplantation by modification of islet surfaces with PEG-lipids; this approach, however, does not suppress immune rejection reactions.

Livers were retrieved from recipient mice after transplantation of control or PEG-islets through the portal vein at $1 \mathrm{hr}$ and 1 day and subjected to histochemical analyses. After control islet transplantation at $1 \mathrm{hr}$ (Fig. 4a), islets were damaged and destroyed in the blood vessel of the liver. Aggregation of red blood cells was observed around destroyed islets, indicating platelet aggregation and blood coagulation on the surface of islets. Cell damage to some islets was also observed after transplantation for 1 day (Fig. 4a). However, in livers of mice transplanted with PEG-islets, most islets were not damaged and remained intact in blood vessels of liver after transplantation for $1 \mathrm{hr}$ (Fig. 4b and c). The same results were observed 1 day after transplantation. These histochemical analyses support the finding that the period of graft survival is prolonged after transplantation of PEG-islets.

\section{Determination of insulin levels in blood after intraportal transplantation of PEG-}

\section{islets}

To determine plasma insulin levels, blood was taken from the subclavian vein 15, 30 and 60 min after intraportal transplantation of control and PEG-islets. When islets 
undergo cell damage, large amounts of insulin from the islets are released into the blood and the blood insulin levels transiently increase [9]. Therefore, the degree of cell damage to islets can be linked to the blood insulin level. In mice transplanted with control islets, high levels of insulin $(\sim 27 \mathrm{ng} / \mathrm{mL})$ were detected $15 \mathrm{~min}$ after transplantation (Fig. 5). The insulin was still observed at 30 and $60 \mathrm{~min}$, although the amount of released insulin gradually reduced. These results indicated that islets were damaged, resulting in the release of large amounts insulin into the blood immediately after transplantation. On the other hand, in mice transplanted with PEG-islets, the release of insulin was considerably suppressed immediately after transplantation $(\sim 7$ $\mathrm{ng} / \mathrm{mL}$ ), indicating that islet cell damage was suppressed by PEG-lipid modification. Thus, this indicates the possibility to improve the biocompatibility of islets by surface modification with PEG-lipids. 


\section{Discussion}

Several groups have attempted to improve graft survival of islets by modification of islet surfaces $[8,9,16,17]$. Byon et al. reported improved graft survival of islets upon covalent bonding of PEG to cell membrane proteins and low dose of immunosuppressive therapy by transplantation under the kidney capsule. However, it is difficult to expect in the current clinical setting that surface modification of islets by PEG would improve transplantation under kidney capsules [18], as clinical islet transplantation to the liver is performed through the portal vein, and islets are exposed to blood components. On the other hand, when islets were transplanted under kidney capsules, cells are not exposed to blood components. The periods of graft survival are quite different in transplantation between these sites [18]. When islets are exposed to fresh blood in the portal vein, the blood coagulation and complement systems are activated, and chemotactic factors, tissue factor, chemokines, and other inflammatory mediators are released during the early phase of transplantation. These factors are induced by instant blood-mediated inflammatory reactions, resulting in graft loss and reduction of graft survival in the liver. Therefore, for future clinical islet transplantation, it is necessary to examine the efficacy of surface modification of islets by transplantation into liver. Several reports have examined the effect on transplantation success when 
transplanting surface modified islets into livers of diabetic animals [8,9]. Contreras et al. reported that the graft survival of porcine islets in livers of NOD-SCID mice was improved by the transfection of an apoptosis suppressing gene and covalent PEGsurface modification [8]. Nilsson et al. reported that the graft survival of porcine islets could be improved by modifying the surface of islets with heparin [9], and found that the heparin coating suppressed blood coagulation and activation of complement systems. In this study, we modified the surface of islets with amphiphilic PEG-lipid and examined the ability of the surface modification to improve graft survival in the liver. Recently we also immobilized bioactive molecules such as fibrinolytic urokinase using amphiphilic polymers to improve graft survival of islets in the liver. We have predicted that the surface modification with PEG and urokinase could suppress the inflammatory and coagulation reactions that mediate islet loss immediately after intraportal transplantation. PEG-lipid was spontaneously anchored to the lipid bilayer of cell membrane through hydrophobic interactions without cytotoxicity. Therefore, it is possible for a thin PEG layer to form on the islet surface without volume increase. PEGlipid has been widely used for the surface modification of liposomes to improve biocompatibility and prolong the circulation time in vivo in drug delivery systems $[19,20]$. Complement activation and platelet aggregation are suppressed by the surface 
modification of liposome with PEG-lipid [21,22]. Our group studied the interaction between serum components and PEG-immobilized substrate by surface plasmon resonance and did not find activation of complement on the PEG-immobilized substrate [23]. In this study, PEG-lipid modified hamster islets were transplanted into livers of STZ-induced diabetic mice through the portal vein. We predicted that complement activation and platelet aggregation could be suppressed in the early phase using the PEG-lipid modified surface, as seen for PEG-liposomes and PEG-immobilized substrate. The graft survival of PEG-islets was significantly prolonged compared with bare islets in livers of diabetic mice (Fig. 3). Reduction of blood insulin level within 60 min after transplantation suggests that the cell damage observed immediately after transplantation could be suppressed by surface modification with PEG (Fig. 5). Platelet activation and complement system activation is thought to be suppressed using islets modified with PEG-lipids, as seen with PEG-liposomes and PEG-immobilized substrate. However, it was not possible to suppress immune-rejection reactions in recipient mice during later phases. Future studies should seek to improve stable and thick polymer layers on the islet surface and reveal the mechanism for prevention of graft loss with PEG modification in vivo and in vitro. 


\section{Conclusions}

A thin PEG layer could form on the surface of islets using PEG-lipids without volume increase and cytotoxicity. Blood coagulation and activation of complement systems, which was induced immediately after intraportal transplantation, could be suppressed using the PEG-lipid modified islets in transplantation, and usually observed islet damage during transplantation was suppressed. Furthermore, the period of graft survival was significantly improved. Together these findings suggest that our approach described here for the improvement of graft survival will be useful in the clinical setting.

\section{Acknowledgements}

This study was supported in part by CREST and JST, and by a Grant-in-Aid for

Scientific Research (A) (No. 18200034) from the Ministry of Education, Culture, Sports,

Science and Technology (MEXT) of Japan and the Ministry of Health, Labor and Welfare of Japan (H20-007). 


\section{References}

(1) Shapiro AM, Lakey JR, Ryan EA, et al. Islet transplantation in seven patients with type 1 diabetes mellitus using a glucocorticoid-free immunosuppressive regimen. N. Engl. J. Med. 2000; 343: 230-238.

(2) Ryan EA, Lakey JR, Rajotte RV, et al. Clinical outcomes and insulin secretion after islet transplantation with the Edmonton protocol. Diabetes 2001; 50: 710719.

(3) Moberg L, Johansson H, Lukinius A, et al. Production of tissue factor by pancreatic islet cells as a trigger of detrimental thrombotic reactions in clinical islet transplantation. Lancet 2002; 360: 2039-2045.

(4) Johansson H, Lukinius A, Moberg L, et al. Tissue factor produced by the endocrine cells of the islets of Langerhans is associated with a negative outcome of clinical islet transplantation. Diabetes 2005; 54: 1755-1762.

(5) van der Windt DJ, Bottino R, Casu A, Campanile N, Cooper DK. Rapid loss of intraportally transplanted islets: an overview of pathophysiology and preventive strategies. Xenotransplantation 2007; 14: 288-297.

(6) Ozmen L, Ekdahl KN, Elgue G, Larsson R, Korsgren O, Nilsson B. Inhibition of thrombin abrogates the instant blood-mediated inflammatory reaction triggered by 
isolated human islets: possible application of the thrombin inhibitor melagatran in clinical islet transplantation. Diabetes 2002; 51: 1779-1784.

(7) Johansson H, Goto M, Dufrane D, et al. Low molecular weight dextran sulfate: a strong candidate drug to block IBMIR in clinical islet transplantation. Am. J. Transplant. 2006; 6: 305-312.

(8) Contreras JL, Xie D, Mays J, et al. A novel approach to xenotransplantation combining surface engineering and genetic modification of isolated adult porcine islets. Surgery 2004; 136, 537-547.

(9) Cabric S, Sanchez J, Lundgren T, et al. Islet surface heparinization prevents the instant blood-mediated inflammatory reaction in islet transplantation. Diabetes 2007; 56: 2008-2015.

(10) Teramura Y, Iwata H. Islets surface modification prevents blood-mediated inflammatory responses. Bioconjugate Chem. 2008; 19: 1389-1395.

(11) Totani T, Teramura Y, Iwata H. Immobilization of urokinase to islet surface by amphiphilic poly (vinyl alcohol) carrying alkyl side chains. Biomaterials 2008; 29: $2878-2883$. 
(12) Miura S, Teramura Y, Iwata H. Encapsulation of islets with ultra-thin polyion complex membrane through poly(ethylene glycol)-phospholipids anchored to cell membrane. Biomaterials 2006; 27: 5828-5835.

(13) Teramura Y, Kaneda Y, Iwata H. Islet-encapsulation in ultra-thin layer-by-layer membranes of poly(vinyl alcohol) anchored to poly(ethylene glycol)-lipids in the cell membrane. Biomaterials 2007; 28: 4818-4825.

(14) Teramura Y, Kaneda Y, Totani T, Iwata H. Behavior of synthetic polymers immobilized on cell membrane. Biomaterials 2008; 29: 1345-1355.

(15) Iwata H, Takagi T, Shimizu H, Yamashita K, Kobayashi K, Akutsu T. Agarose for bioartificial pancreas. J. Biomed. Mater. Res. 1992; 26: 967-977.

(16) Lee DY, Park SJ, Nam JH, Byun Y. A new strategy toward improving immunoprotection in cell therapy for diabetes mellitus: long-functioning PEGylated islets in vivo. Tissue Eng. 2006; 12: 615-623.

(17) Yun Lee D, Hee Nam J, Byun Y. Functional and histological evaluation of transplanted pancreatic islets immunoprotected by PEGylation and cyclosporine for 1 year. Biomaterials. 2007; 28: 1957-1966. 
(18) Goto M, Johansson H, Maeda A, Elgue G, Korsgren O, Nilsson B. Low molecular weight dextran sulfate prevents the instant blood-mediated inflammatory reaction induced by adult porcine islets. Transplantation 2004; 77: 741-747.

(19) Zalipsky S. Functionalized Poly(ethylene glycol) for preparation of biologically relevant conjugates. Bioconjugate Chem 1995; 6: 150-165.

(20) Klibanov AL, Maruyama K, Torchilin VP, Huang L. Amphipatic poly(ethylene glycol)s effectively prolong the circulation time of liposome. FEBS Lett 1990; 268: 235-237.

(21) Bradley AJ, Devine DV, Ansell SM, Janzen J, Brooks DE. Inhibition of liposomeinduced complement activation by incorporated poly(ethylene glycol)-lipids. Arch. Biochem. Biophys. 1998; 357: 185-194.

(22) Chiu GN, Bally MB, Mayer LD. Effects of phosphatidylserine on membrane incorporation and surface protection properties of exchangeable poly(ethylene glycol)-conjugated lipids. Biochim Biophys Acta. 2002; 18: 37-50.

(23) Arima Y, Toda M, Iwata H. Complement activation on surfaces modified with ethylene glycol units. Biomaterials. 2008; 29: 551-560. 


\section{Figure Captions}

Figure 1. (a) Chemical structure of PEG-conjugated DPPE (PEG-lipid). (b) Confocal laser scanning images of FITC-PEG-lipid-modified islets from hamsters. PEG-lipid can be introduced on the surface of islets by hydrophobic interaction. The hydrophobic lipid portion of PEG-lipid was spontaneously incorporated into the lipid bilayer membrane of islets.

Figure 2. Assessment of insulin release in glucose stimulated islets. The amounts of insulin secreted from PEG-islets (black bars) and control bare islets (white bars) in response to glucose concentration changes $(0.1,0.3,0.1 \mathrm{~g} / \mathrm{dL})$ were determined by ELISA. Results are expressed as mean $\pm \mathrm{SD} ; n=3$.

Figure 3. Changes in non-fasting blood glucose levels of streptozotocin-induced diabetic Balb/c mice after intraportal transplantation of (a) 500 control islets $(n=9)$, (b) 1000 control islets $(n=4)$, (c) 500 PEG-islets $(n=6)$, or (d) 1000 PEG-islets $(n=5)$. Graft failure was defined as two consecutive plasma glucose determinațons 00 $\mathrm{mg} / \mathrm{dL}$. 
Figure 4. Histochemical analysis of (a) control islets and (b) PEG-islets transplanted into the liver of diabetic mice through the portal vein by hematoxylin-eosin staining, and (c) PEG-islets by insulin staining. These grafts were retrieved at $1 \mathrm{hr}$ and 1 day post-intraportal transplantation. The graft obtained $1 \mathrm{hr}$ after transplantation was also stained for insulin and the nucleus (Hoechst 33342).

Figure 5. Changes in the blood insulin levels of diabetic mice after intraportal transplantation of control islets (closed circles, $n=7$ ) or PEG-islets (open circles, $n=7$ ). The blood insulin levels were determined by ELISA. 
(a)

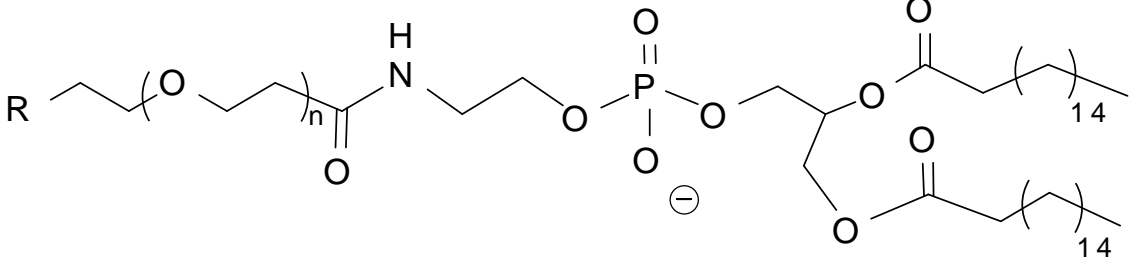

$$
\begin{aligned}
& \text { R: Cystein-maleimide }
\end{aligned}
$$

(b)
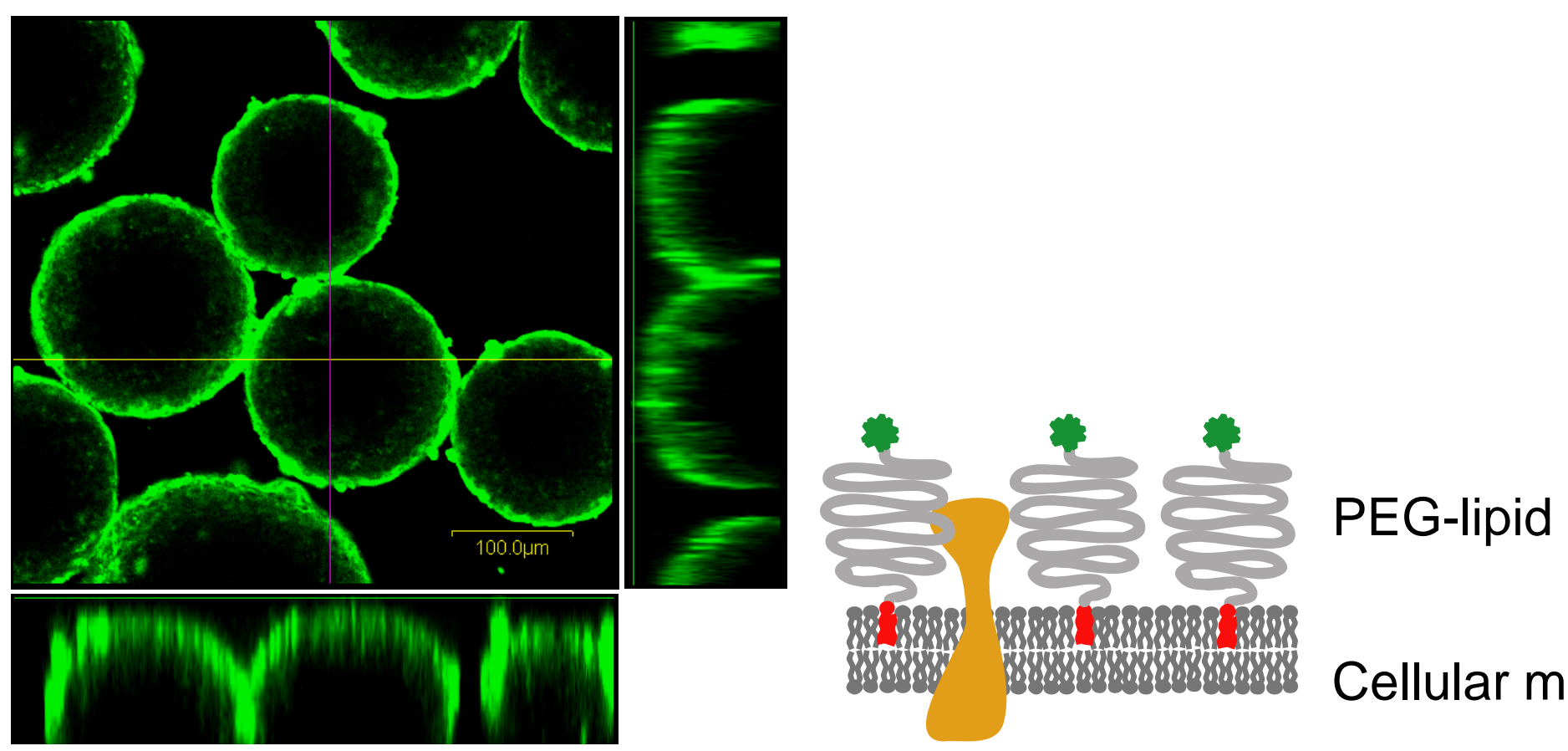

Figure 1 


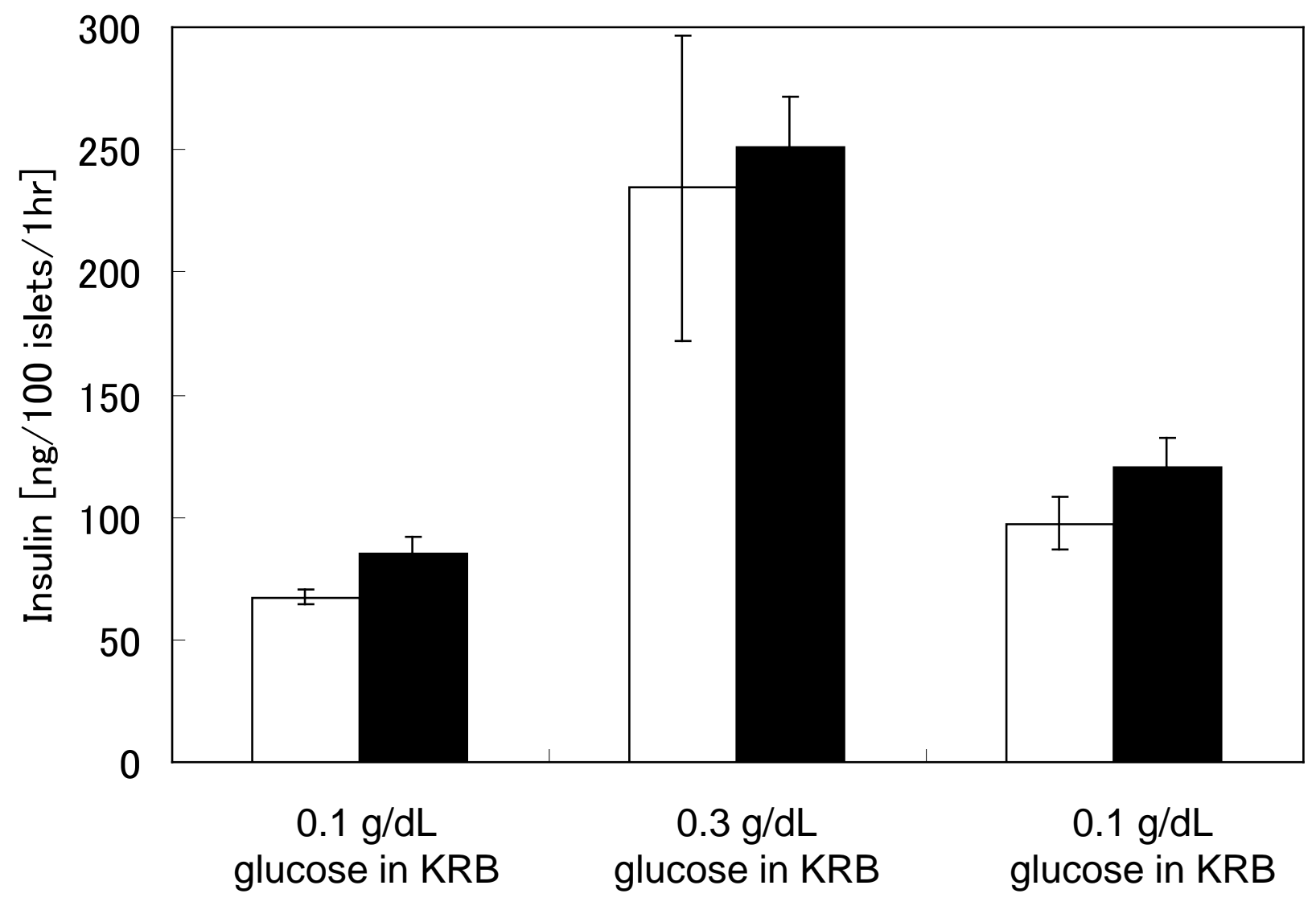

Figure 2 


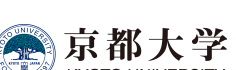

A Self-archived copy in
Kyoto University Research Information Repository

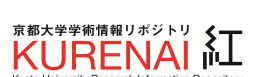

(a)

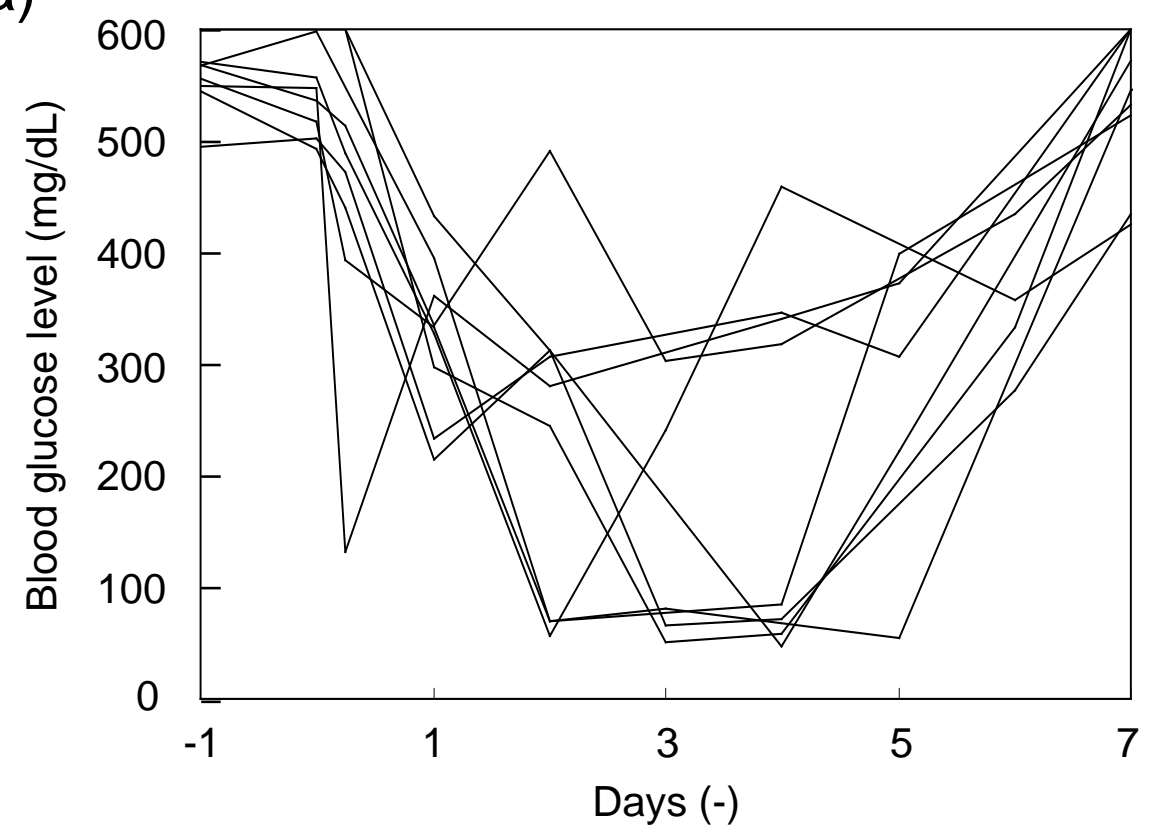

(c)

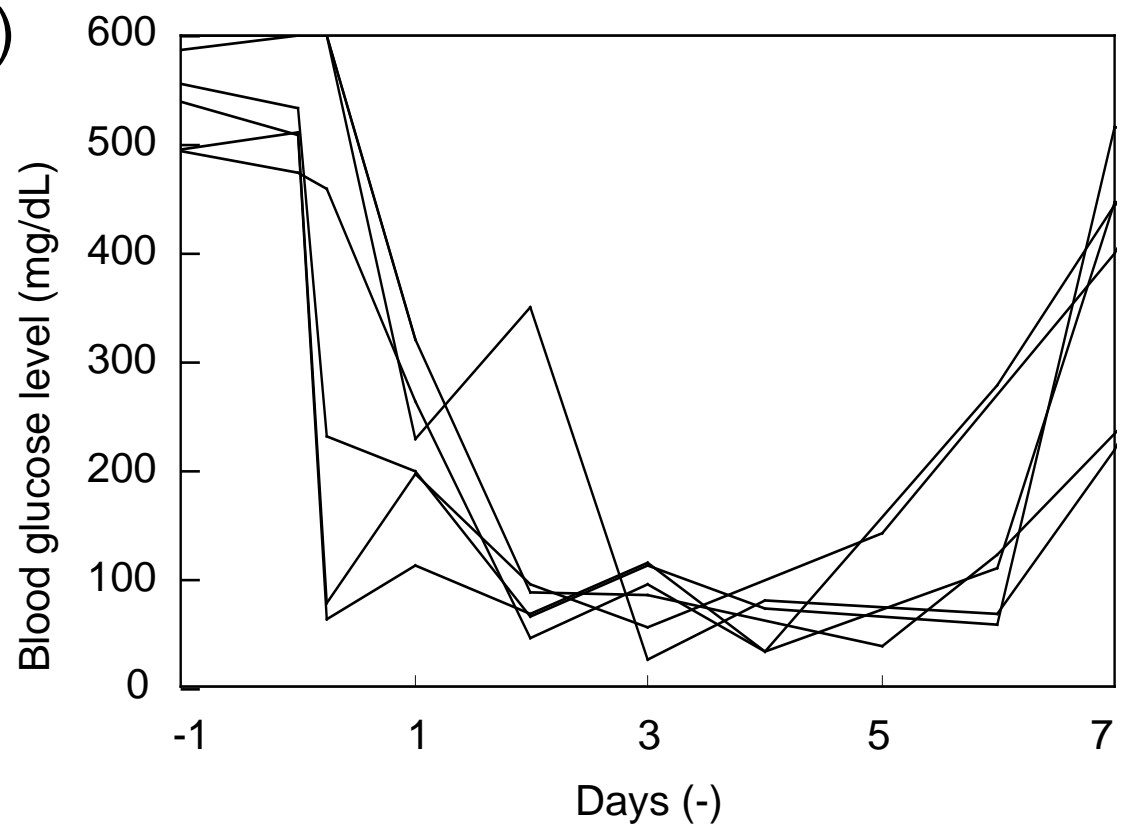

(b)

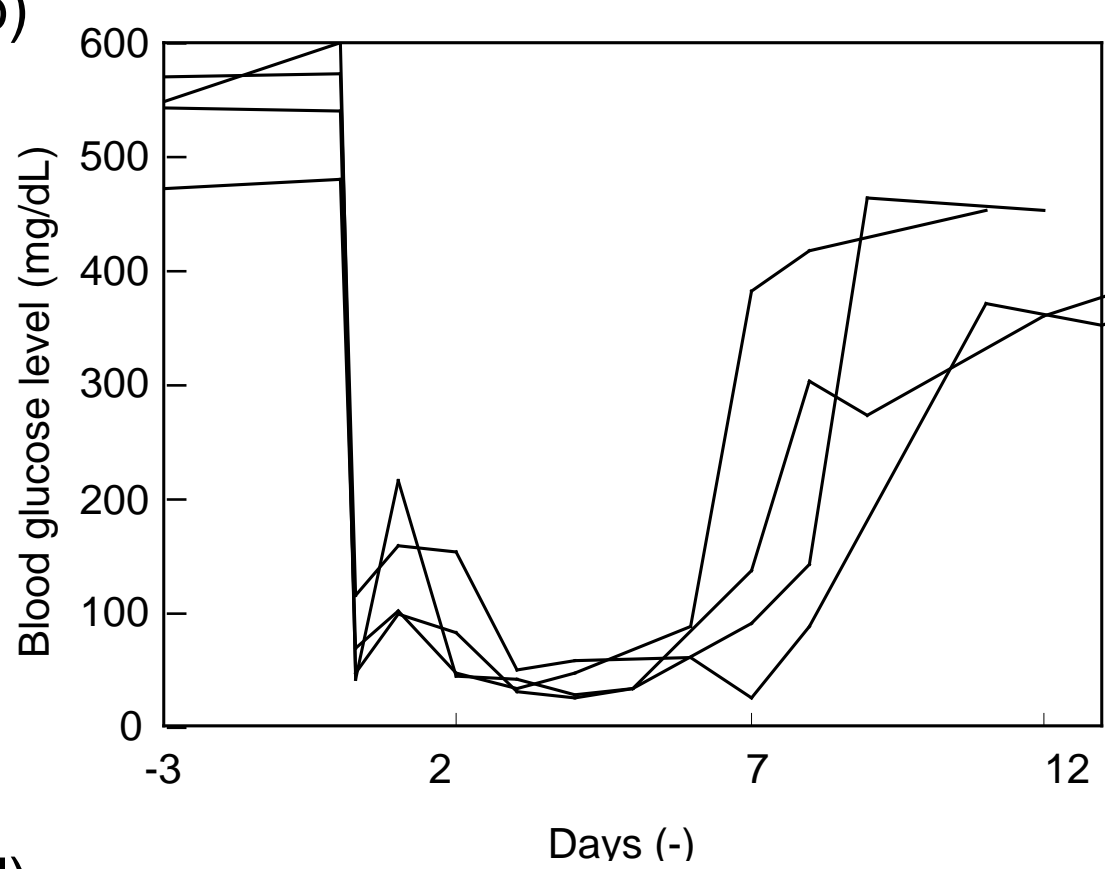

(d)

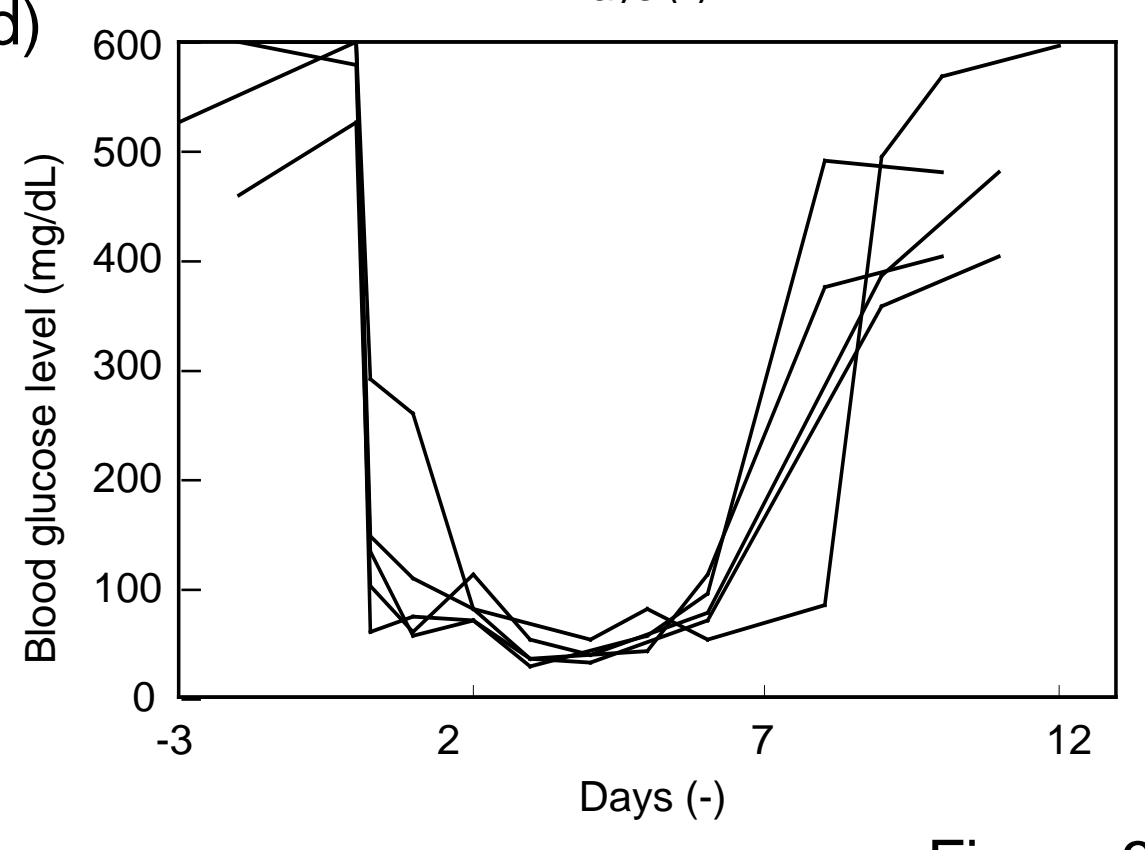

Figure 3 
(a)
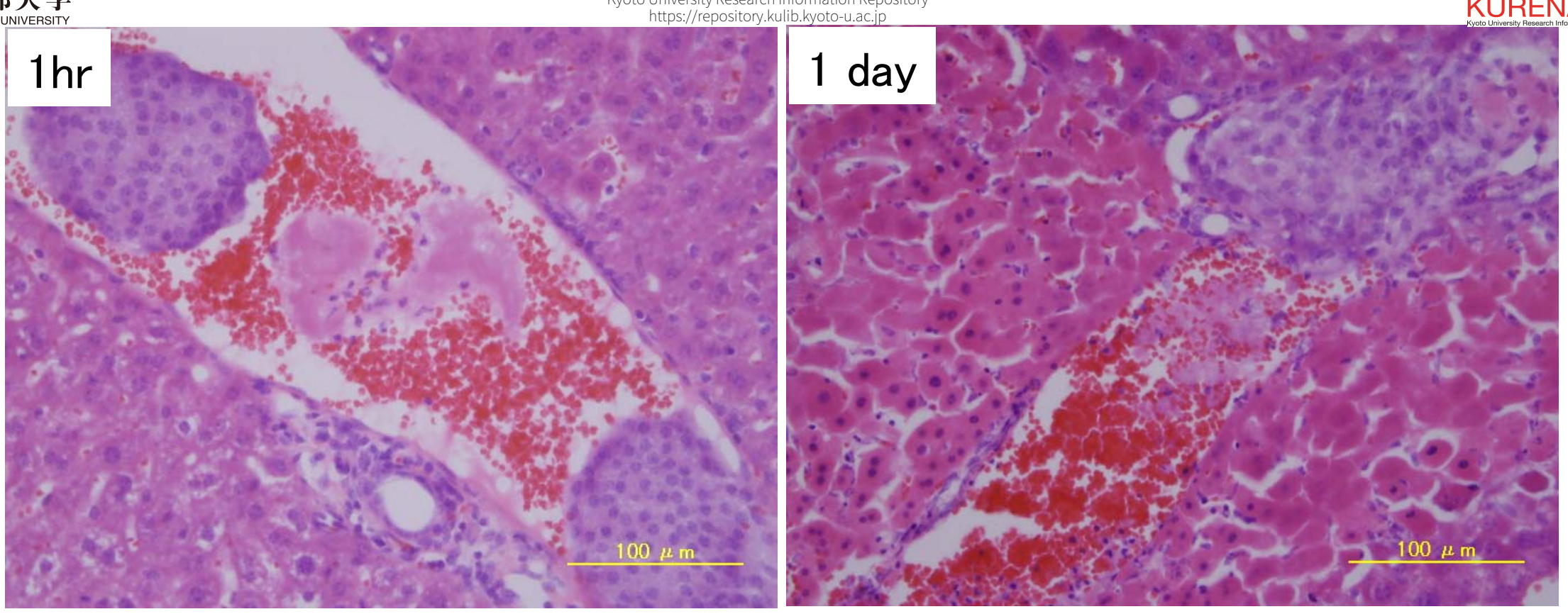

(b)

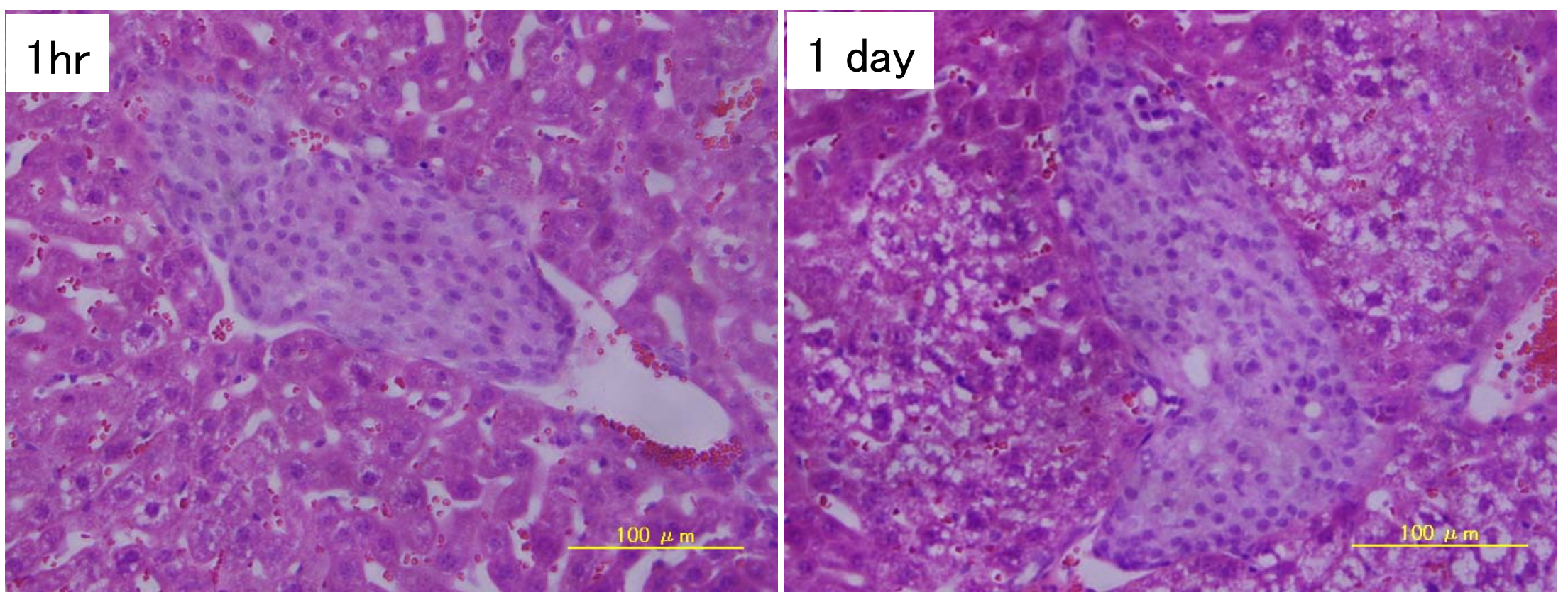

Figure 4 
(C)

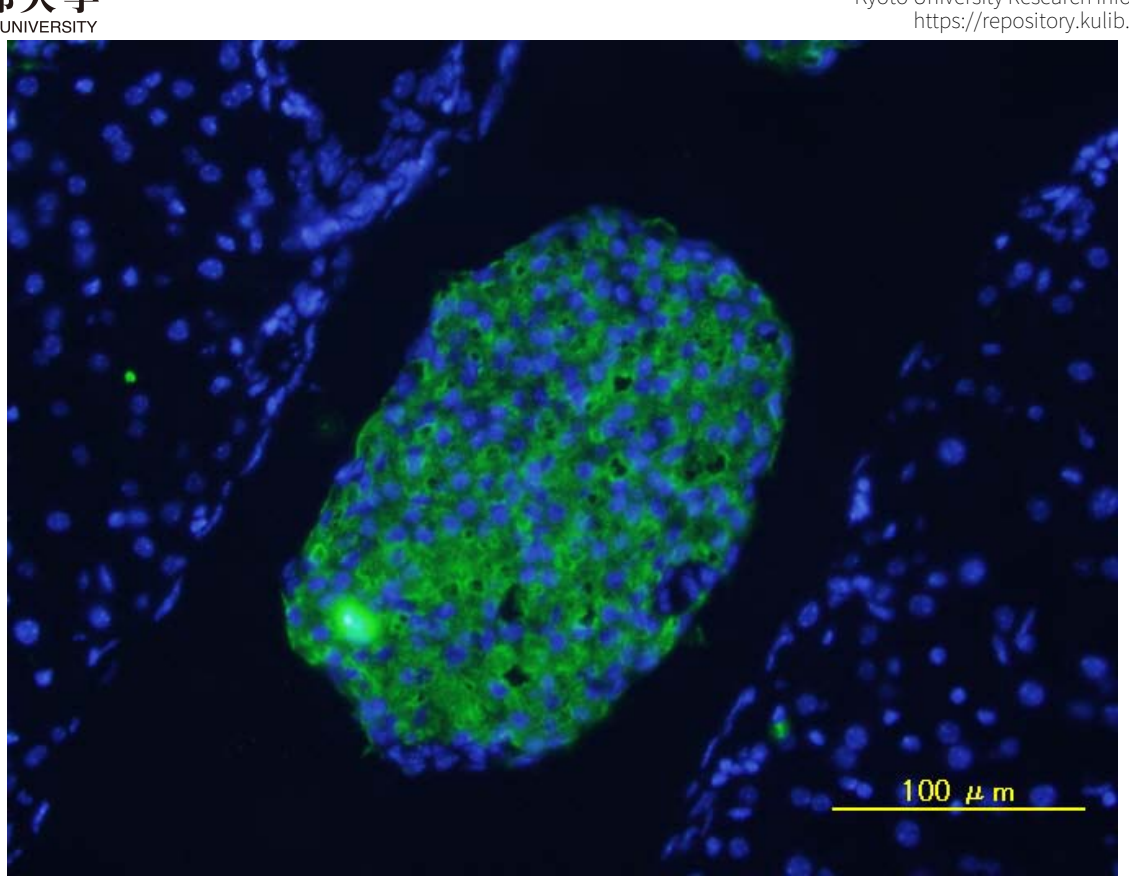

Figure 4 


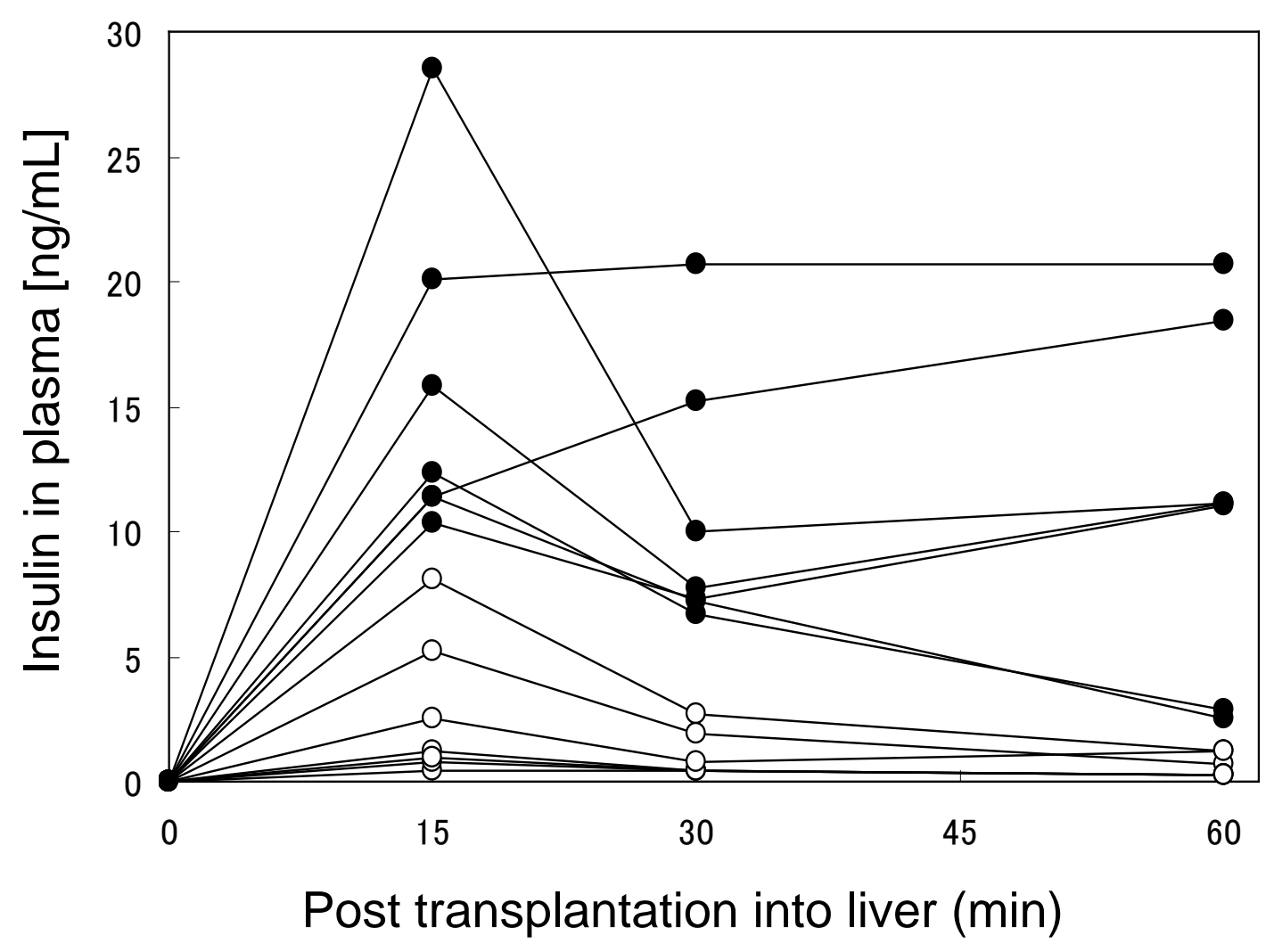

Figure 5 
Table 1. Graft survival days after transplantation.

$\left.\begin{array}{llll}\hline & \text { Number } & \text { Graft survival days } & \text { Mean } \pm \text { SD } \\ \hline \text { control islets } & 500 & 0,0,0,1,2,3,3,3,4 & 1.8 \pm 1.6 \\ & 1000 & 6,7,8,9 & 7.5 \pm 1.7 \\ \text { PEG-islets } & 500 & 4,4,5,5,6,6 & 5.0 \pm 0.9\end{array}\right] p<0.01$

Graft failure was defined as two consecutive plasma glucose determinations $\geq 200 \mathrm{mg} / \mathrm{dL}$. 